VS42-03

\section{Shift fields: A new approach to refinement using non-atomic parametrizations}

Kevin Cowtan ${ }^{1}$, Jon Agirre², Stephen Metcalfe ${ }^{2}$

1. Department of Chemistry, University of York, United Kingdom

2. University of York, York, United Kingdom

email: kevin.cowtan@york.ac.uk

Model refinement is a key step in crystallographic structure solution, allowing an atomic model to be optimised to best explain the crystallographic observations and reconstruct the missing phases. The scattering density is usually described in terms of atomic parameters, however in macromolecular crystallography the resolution of the data is often insufficient to determine the values of those parameters for individual atoms. Stereochemical and geometric restraints are used to provide additional information, but produce interrelationships between parameters which slow convergence.

These problems arise because atoms are small and their scattering density hard to see at low resolution. One solution with a historical precent in existing methods is to uncouple the model from the atoms by attaching the model parameters to regions of the electron density map rather than to atoms. These parameters can move the density or change the local temperature factor to better explain the structure factors. The parameters may be optimized at higher or lower resolutions by simply varying the volume of the region used to determine the parameter shifts, creating a smooth distortion of the model which partially mitigates the need for stereochemical restraints. The resulting method has a wide range of convergence and can be applied at low resolution, which also makes the calculation many times faster than comparable methods. Potential applications include massive screening of molecular replacement models, initial refinement of models with domain motions, and potentially the use of electron density from other sources such as cryo electron microscopy (cryoEM) as a refinement model.

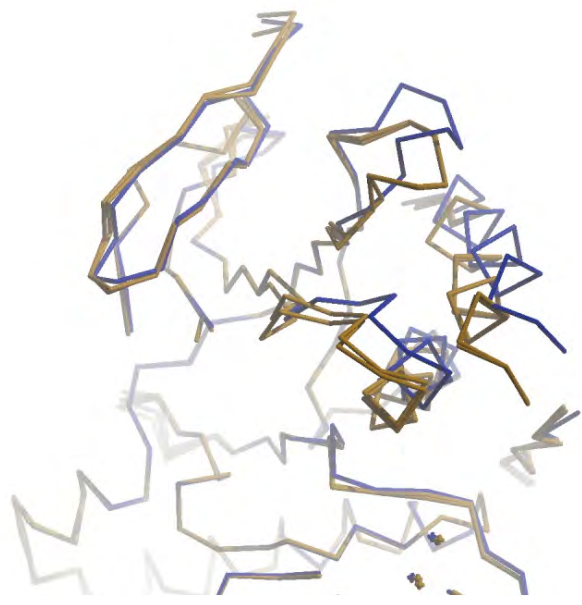

References:

Cowtan, K., \& Agirre, J. (2018). Macromolecular refinement by model morphing using non-atomic parameterizations. Acta Crystallographica Section D: Structural Biology, 74(2), 125-131.

Keywords: refinement, molecular replacement, macromolecular crystallography
MS42-04

\section{Building atomic models into electron- microscopy maps with ARP/wARP version 8.0}

Grzegorz Chojnowski ${ }^{1}$, Philipp Heuser ${ }^{1}$, Joana Pereira ${ }^{1}$, Victor Lamzin $^{1}$

1. EMBL Hamburg, Hamburg, Germany

email: gchojnowski@embl-hamburg.de

Recent developments in instrumentation and data processing techniques enabled determination of cryo-electron microscopy (cryo-EM) maps at resolutions previously attainable exclusively by macromolecular crystallography (MX). Indeed, the number of cryo-EM reconstructions at a resolution better than $4.0 \AA$ deposited in Electron Microscopy Databank, which in principle allows for automated de novo model building, is growing fast.

The ARP/wARP software has, for over twenty years, provided continuously advancing tools for MX structure determination [1]. Here we present recent developments of ARP/ wARP that enable building atomic models of proteins and protein-nucleic acid complexes into cryo-EM maps at a resolution better than $4.0 \AA$. An automated input map pre-processing has been introduced to assure desired level of map sharpening. The main-chain tracing routines for proteins and nucleic acids have been advanced to yield more reliable models with improved local stereochemistry [2] and their applicability has been specifically extended to the cryo-EM maps. A new protein sequence-docking algorithm has been developed building up on the fragmentation tree concept [3] and on graph theory approaches. While having been specifically developed for cryo-EM, the new sequence docking also provides improved performance for low-resolution MX cases. Finally, the implementation of a new method for building poorly defined loops in cryo-EM maps has helped reduce the fragmentation of the built model.

Initial tests on deposited cryo-EM maps have been particularly encouraging, and the obtained results will be presented and discussed.

The module for the interpretation of EM maps has been made available with the most recent ARP/wARP 8.0 release and access to remote computation is provided with the redesigned web server at https://arpwarp.embl-hamburg.de

References:

[1] Langer et al. (2008). Nature Protoc. 3, 1171-1179.

[2] Pereira J, Lamzin VS. (2017). IUCrJ 4, 657-670.

[3] Langer et al. (2012). J Mol Biol. 419, 211-222.

Keywords: Model Building, Refinement, macromolecular crystallography, cryo-EM 